\title{
Nokia's IN solution for fixed and cellular networks
}

Pekka Lehtinen

Markus Warsta

Nokia Telecommunications

P.O. Box 33

02601 Espoo, Finland

\section{INTRODUCTION}

The development of Intelligent Networks (IN) was started by Bellcore in USA more than ten years ago in order to support the Regional Bell Operating Companies in the new deregulated telecommunications environment. The original goal was to enable the network operators to effectively introduce and manage new services by means of a centralized database in a Service Control Point (SCP).

In the past years, several IN specifications have been developed, including IN 1, AIN Rel.0 and AIN Rel.1. The Advanced Intelligent Network Release 1 (AIN Rel.1) of Bellcore contains a service-independent standard interface for the queries sent from the Service Switching Points (SSPs) to the SCP.

The International Telecommunications Union (ITU) has worked out its Intelligent Network Application Part (INAP) specifications for the SSP-SCP interface, the so-called Capability Set 1 (CS.1). The European Telecommunications Standards Institute (ETSI) has completed its corresponding ETSI CS.1 Core specifications in 1993. It is expected that the CS.1 recommendations will be adopted world-wide. This would offer open interfaces between the SSP and SCP resulting in vendor independence.

Signalling System No.7 (SS7), fault-tolerant computing, on-line transaction processing, and today's database technology have enabled the development of efficient IN elements. This paper presents Nokia's IN solution. 


\section{FUNCTIONAL ARCHITECTURE OF INTELLIGENT NETWORKS}

The main elements of the IN architecture of the ITU and ETSI are:

- Service Switching Point (SSP),

- Service Control Point (SCP),

- Service Management System (SMS),

- Service Creation Environment (SCE), and

- Intelligent Peripheral (IP).

The SSPs and SCPs are interconnected by the SS7 network. The SMS and SCP are connected by data communication protocols.

SSP provides the end-users an access to the IN services. It contains trigger points for detecting service access codes and sending service requests to the SCP. It also performs the required network-related operations based on the information received from the SCP.

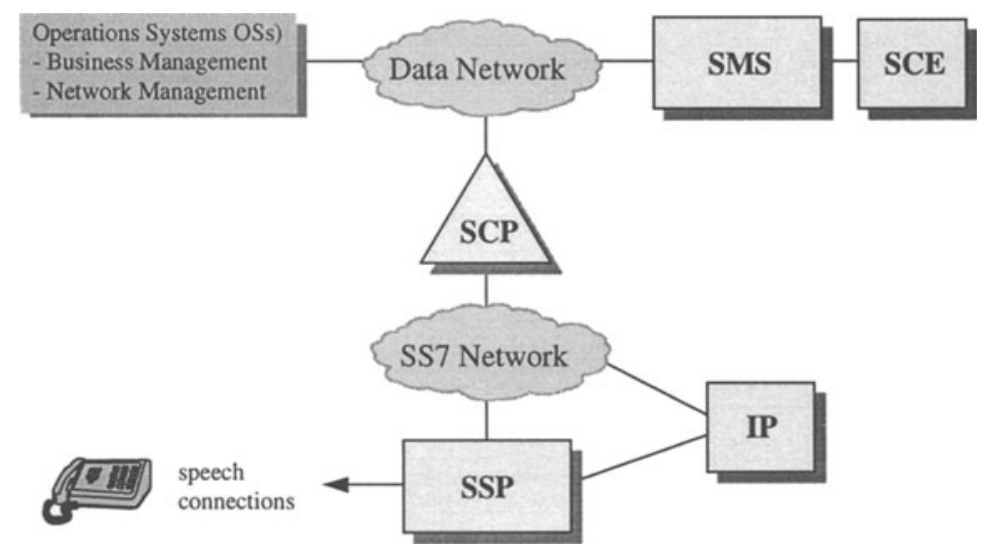

Figure 1. Functional Architecture of the IN.

In the SCP, Service Logic Programs (SLPs) contain the control logic of the service functionality, and the database contains the required information.

SMS includes IN management applications and capabilities to control the service providers. It is used

- to download the service database to the SCPs,

- to provide interfaces with service subscribers and service providers for the management of service parameters, and

- to gather measurement data.

SCE contains tools for the development of SLPs. The software development can be carried out e.g. in UNIX workstations running the SCE. 


\section{NOKIA'S IN APPROACH}

\subsection{Objectives}

Service complexity, signalling capabilities, network coverage and revenue sharing are the variable factors, that determine the requirements of each customer. Nokia offers solutions for different customer environments. A good solution meets the customer's current economic and technical needs and allows an evolution towards the future network architecture.

Network operators are facing new challenges due to new trends of customer expectations, regulatory environment and information technology. However, the limitations of the current networks must be taken into account. The migration to a new network environment and new services must be smooth in order to ensure the protection of investments.

The objective of Nokia's IN approach is to offer solutions, that meet the following market drivers and network operators' requirements:

- reaction to the customers' needs and new market opportunities,

- competition in the deregulated environment,

- introduction of open systems environment,

- generation of revenue from the existing investment,

- low initial investment,

- low investment in reusable equipment and a long-term plan of new equipment,

- end-users' awareness of grade-of-service and availability.

A variety of signalling systems are in use, including decadic and multi-frequency signalling systems. Some networks are widely using the SS7, while others are in the middle of a transition towards the SS7. The network operators' main concern is often the creation of an IN service offering environment within the confines of the network signalling capability.

In co-operation with Hewlett-Packard, Nokia is implementing a combination of open computer and telecommunications technology for providing Intelligent Network capabilities to fixed and mobile network operators. This IN architecture enables a unified solution to fixed and mobile networks in accordance with the new ETSI CS.1 Core specification.

\subsection{Centralized IN Architecture}

Nokia has developed a general service-independent SSP-SCP interface specification based on the CS.1 and the Transaction Capabilities Application Part (TCAP).

The main elements of Nokia 's solution for a centralized IN architecture are:

- DX 200 SSP (including the IP functionality),

- Unix SCP,

- Unix SMS.

Nokia's DX 200 switching system is highly modular. It is based on a distributed call control architecture. These characteristics facilitate a flexible introduction of the IN features and other new capabilities. 


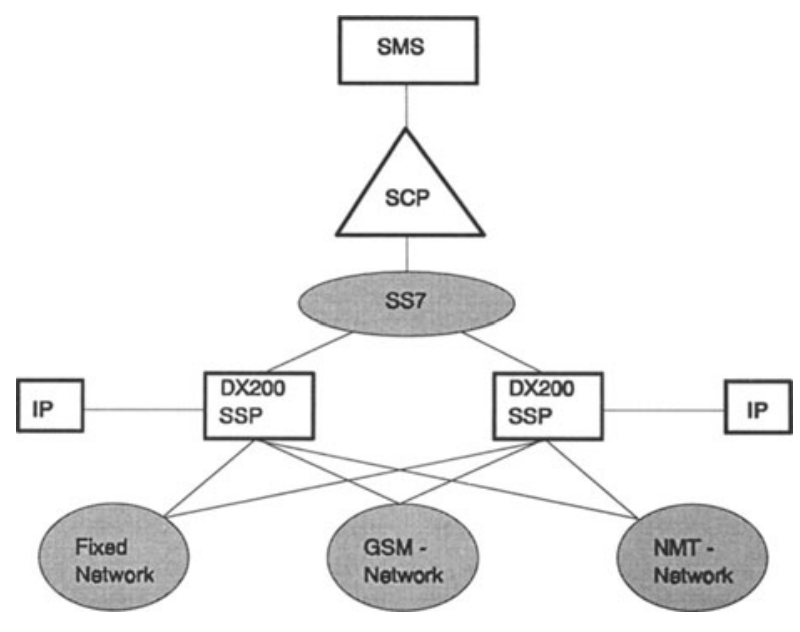

Fig. 3 Centralized IN Architecture

\section{200 SSP}

DX 200 SSP can be configured for the IN services only, but it can also contain the functionality of a normal exchange. The capacity can be easily upgraded to a very high level by adding processing units.

DX 200 SSP can perform Global Title translation. Thus, it can send queries to multiple SCPs. This is useful, if the SCCP function is not generally available in the network. An important aspect is the ability to add new triggers or change the parameters of the triggers in the SSP by using Man-Machine Language (MML) commands.

DX 200 SSP has a built-in IP functionality for sending announcements and collecting DualTone-Multi-Frequency (DTMF) digits as requested by the SCP. In addition, an external voice announcement machine can be installed.

The first release of the DX 200 SSP was based on the Bellcore AIN Rel.1 specifications supporting the functionality needed for the freephone, premium rate, personal number, calling card and VPN services. Subsequent releases of the SSP also meet the ETSI CS.1 Core requirements.

\section{Unix SCP}

The Unix-based SCPs compliment a centralized SCP by decentralizing the control intelligence of local-type services and mass calling applications at lower levels in the network. It is based on the CS.1 specifications.

The Unix SCP is based on the standard Unix platform of Hewlett Packard. The use of standard high-volume Unix computers based on the RISC technology ensures a declining trend of the price-performance ratio. Fault tolerancy is achieved by a duplicated processor. The processing capacity is 400 transactions per second. It can be increased by adding one or more 
mated SCPs which have an identical database. The maximum size of the service database is 500 MBytes of Random Access Memory.

\section{Unix SMS}

The Unix SMS contains the master database of the underlying SCPs. Database changes are automatically propagated from the SMS to the SCPs. If end-users are allowed to make changes in the service database, e.g. C-number updates, these changes are also propagated to the SMS and further on to the mated SCPs.

The SMS includes applications for service management, configuration management, fault management, and performance management. SMS is used to gather service-traffic-related measurement data from the SCPs.

The user interface of the SMS is based on a local area network (LAN). Unix-based workstations connected to the LAN include a variety of tools for the operating personnel to perform operations and maintenance tasks. They also provide a Service Creation Environment (SCE) to the network operator for the development of new services and the modication of the existing ones.

\section{Unix SCE}

In the service creation process, several high-level steps can be identified, such as service definition, specification, development, verification, deployment, and version control.

SCE can be used during the full service creation process. It is implemented by HP and is running on Unix workstations, which can be connected to the SMS via a LAN. It contains the following components:

- a graphical interface for rapid service creation (editing, validation),

- a simulated SMS for initialising the database with structural information of the subscribers' data records,

- a software-simulated SCP,

- a software-simulated SSP, with the ability to simulate the call traffic.

\section{SSP-SCP Interface}

The INAP between the SSP and SCP is based on the ETSI CS.1 specifications. The application messages are carried in TCAP messages. The SCCP and the Message Transfer Part (MTP) constitute the lower protocol levels.

INAP supports several advanced service features, including

- call-origin-based blocking or filtering (based on area code, country code, or A-subscriber's number),

- routing, depending on additional dialled digits,

- routing, depending on call characteristics (e.g. requirement for a digital connection),

- follow-me diversion,

- C-number update,

- rerouting in the busy and no-answer cases,

- follow-on calls in the case of the Calling Card service (i.e. sequence call, extension of the INAP),

- charging options (e.g. alternate billing, billing code, or tariff, extension of the INAP). 


\subsection{Decentralized IN Architecture}

In many telecommunications networks, the SCPs are centrally located and the SSPs are distributed at the transit exchange level. This solution has a number of advantages:

- The implementation and deployment of new services is fast.

- The IN service management is centralized in a few network nodes.

- The IN architecture is well suited for nationwide services, such as network-wide routing and call-distribution services.

On the other hand, some services (e.g. televoting) generate heavy traffic. This may cause overload problems, if the traffic is concentrated on a few SSPs and a centralized SCP.

A number of services are not suited for implementation in a centralized IN architecture. Some services are strictly local by nature (e.g. incoming call screening), or the response time delay of a centralized SCP is unacceptable, or a centralized SCP causes capacity limitations. In addition, some services require more information about the caller than what can be provided by the SSP at the transit level. Due to these requirements, it is obvious that the SSP functionality must be migrated down to the local exchange level as far as SS7 is available. From the viewpoint of the SCP, this evolution trend has the following advantages:

- short response time to end-users,

- possibility to increase the processing capacity according to customer requirements,

- capability to customize services for local use.

Several SCPs may be needed in order to offer a sufficient coverage of the network. The SCPs can be allocated to networks, regions, or services depending on the network, service characteristics, and customer requirements.

A great benefit of the above mentioned IN architecture is the low cost of implementing a true transitional IN architecture, including the reuse of service applications. This offers the network operator an opportunity to take advantage of the SS7 network without neglecting other parts of the network.

Depending on the signalling system, it may be possible to transfer the A-subscriber's identity from a local exchange to the SCP. The A-subscriber's validation in the SCP enables the creation of a charging record for each identified subscriber. In particular, Premium Rate service with various charging functions can be provided. A number of other services can be provided to the subscribers who are connected to the SCP.

\subsection{Customer Implementations}

Nokia has been in the forefront of IN development, providing advanced services based on a pre-IN architecture and subsequent true IN solutions based on open service-independent interfaces.

An AIN Rel.1-based IN solution has been delivered to Telecom Finland. The SSP exchanges are based on the DX 200 switching platform. In the first phase, Freephone, Premium Rate, Personal Number, and Calling Card Validation services were implemented.

Nokia has pioneered the development of the service-independent general SSP-SCP interface. Based on the AIN Rel.1 specifications, the INAP protocol, SS7 and TCAP have been implemented. In spite of its many advantages, the AIN Rel.1 specifications are 
incomplete. Adaptations and additions were also necessary due to the differences between the telephone networks in USA and Finland in the area of numbering schemes, charging procedures, and signalling capabilities.

Nokia has delivered a DX 200-based Service Switching and Control Point (SSCP) to Tele 2, a Swedish network operator. In this case, the AIN Rel.1 specification is used in the interface between the Service Switching Function (SSF) and the Service Control Function (SCF) integrated into the same exchange. The Application Service Elements (ASEs) are transferred via a high-speed message bus, instead of using the SS7. A good example of the capabilities of the DX 200 switching platform is that the same DX 200 SSP is also used as an international switch.

The DX 200 SSCP of Tele 2 provides access validation services that enable the end-users to make international calls via the network of Tele 2 , even if the terminal is not directly connected to this network. The SSCP contains a subscriber database, which is used to validate the identity of the calling station and its rights to make the call.

The first contract to deliver an open IN solution based on the Nokia-HP IN architecture has been signed with Tele 2 . This will enable Tele 2 to widen the service offering.

\subsection{Further IN Development}

It is evident, that the IN standardization activities will continue. Many issues are subject to further standardization, including global IN services, service portability, service feature interworking, and the interfaces between the SCP, SMS and SCE .

It is foreseen, that there is a growing market for services, that provide the mobility of persons and services in addition to terminal mobility. This implies a need to receive and make calls by using a personal number across multiple fixed and cellular networks. In addition, the same personalized service features should be available to all the end-users, irrespective of their terminal. There are also many cellular-network-specific requirements, such as a need to know the location of the calling mobile terminal in the case of an IN-based emergency call.

The functional integration of IN mobility services arises several questions. How to interchange customer profile and call history information between the service nodes? What method can be used to access the location registers? Nokia's strategy is to have a common IN platform for the fixed and cellular network elements in order to ensure future-proof solutions to the evolving mobility requirements.

It is obvious, that SSF will become an optional part of the DX 200 switching platform, including exchanges in fixed and cellular networks. This capability will offer the network operator a great flexibility regarding the network configuration and the customer-specific service options.

Integration of the SSF into a mobile services switching system will provide an environment, where the combined capabilities of a GSM/DCS network and IN can be fully utilized. The importance of openness is emphasized by the operators. It can be achieved by designing the SSP-SCP interface and the call model in accordance with the ETSI CS.1 Core specification. 